\title{
Resistive Sensor for Short High-Power Microwave Pulse Measurement in Millimeter Wave Range
}

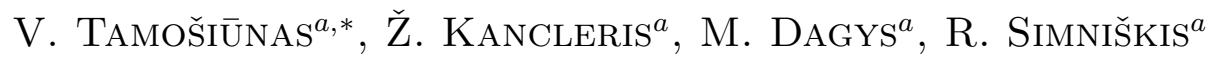 \\ AND F.J. AGEE ${ }^{b}$ \\ ${ }^{a}$ Semiconductor Physics Institute, A. Goštauto 11, Vilnius, Lithuania \\ ${ }^{b}$ AFOSR/NE, 801 N. Randolph St., Arlington, VA 22203, USA
}

We report on the numerical investigation of the resistive sensor for the 78-118 GHz range. A three-dimensional finite-difference time-domain method was applied to simulate the wave propagation within a waveguide segment with the semiconductor sensor attached to a wider wall of the wave-guide. The electric field distribution, voltage standing-wave ratio and the average electric field dependences on frequency have been determined for several sets of dimensions and specific resistances of the sample. It is demonstrated that a proper selection of the dimensions and specific resistance of the sample can compensate the waveguide dispersion and the decrease of the electron heating effect with frequency. Therefore, a nearly constant sensitivity of the sensor can be obtained for the entire frequency range.

PACS numbers: 72.20.Ht, 72.30.+q, 85.30.De

\section{Introduction}

Generation of microwaves in a millimeter range progressed largely in the last decade. High power microwave (HPM) pulsed sources generating subnanosecond duration hundred of $\mathrm{kW}$ and even MW pulses are either available in laboratories or being under development now [1,2]. This imposes new requirements for the diagnostics of such pulses since the standard measurement methods using semiconductor diodes are of little use in this case. One of the possible solutions might be a resistive sensor (RS) the performance of which is based on the electron heating effect in $n$-Si. The waveguide type RS shows itself as an attractive device for HPM pulse measurement in a centimeter wave region [3]. It measures HPM

*corresponding author; e-mail: vincas@pfi.lt 
pulses directly, it is resistant to large power overloads and demonstrates a very good long-term stability. The RS can produce an output signal on the order of a few tens of volts without any amplification circuit. It can be done sufficiently fast to measure nanosecond duration HPM pulses. The RS can be employed in the millimeter range as well, since the electron heating effect in $n$-Si disappears in THz region [4]. Unfortunately, a simple downscaling of the successful RS design at low frequency in order to obtain a resistive sensor for the millimeter wave region is difficult if not impossible to realize due to numerous mechanical manufacturing problems. In the present paper we propose a new type of waveguide RS that is suitable for HPM pulse measurement in the millimeter wave region.

\section{Concept of the sensor}

The sketch of proposed RS is shown in Fig. 1. It is seen that it consists of two sensing elements mounted in a close proximity to each other. Upper contacts of the sensing elements are connected in short with thin metal foil. The lower

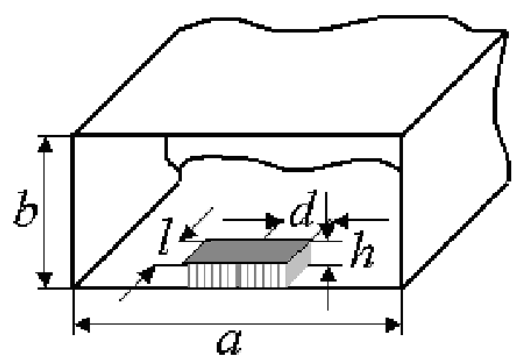

Fig. 1. Cross-sectional view of the proposed RS in the waveguide.

contact of one of the sensing elements is grounded while the other one is isolated and is used for the RS feeding and output signal measurement. Actually, the sensing elements are connected in series in respect of the dc circuit but in parallel in respect to the microwave electric field. Electric field of microwave pulse heats electrons in the sensing elements, their resistance changes and by measuring this resistance change, microwave pulse power in the waveguide is determined.

In this contribution we report on the sensor optimized for the waveguide dimensions of $a=2.4 \mathrm{~mm}, b=1.2 \mathrm{~mm}$ and $78-118 \mathrm{GHz}$ frequency range. The electromagnetic wave propagating in the section with semiconductor sample shown in Fig. 1 was modeled for several sets of dimensions and specific resistance of the sensing elements. The choice of their parameters is restricted by the practical requirements for the RS design. Total resistance of the RS should be less than $50 \Omega$ and the voltage standing wave ratio $<1.2$. 


\section{Dependence of the RS sensitivity on frequency}

The dependence of the output signal of the RS on frequency $f$ can be expressed in the following way [3]:

$$
U_{\mathrm{S}}(f)=U_{0} \beta^{*}(f) E^{2}(f) .
$$

Here $U_{0}$ is the dc voltage drop on the RS, $\beta^{*}$ is a warm electron coefficient defining the deviation of the current-voltage characteristic from Ohm's law and $E^{2}(f)$ is an average of square of the electric field amplitude within the sensor.

A warm electron coefficient is dependent on a frequency and this dependence can be expressed like:

$$
\beta^{*}(f)=\beta\left\{1+2 /\left[1+\left(2 \pi f \tau_{\varepsilon}\right)^{2}\right]\right\} / 2,
$$

where $\beta$ is the warm electron coefficient in the dc electric field and $\tau_{\varepsilon}=2.9 \times 10^{-12} \mathrm{~s}$ is a phenomenological energy relaxation time for $n$-Si in room temperature. In addition, the frequency dependant relation between the amplitude of $E_{y}$ component of $H_{10}$ wave in the center of the empty waveguide and the transmitted power $P$ has to be taken into account when designing a sensor for power measurement applications:

$$
E_{0}^{2}(f)=\frac{480 \pi}{a b \sqrt{1-\left(f_{\mathrm{c}} / f\right)^{2}}} P,
$$

where $f_{\mathrm{c}}$ is cut-off frequency of the waveguide.

As could be seen from (1)-(3) the output signal of the RS decreases with frequency. Thus the increase of the average electric field in the RS is desirable to compensate the decrease of the sensitivity.

\section{Simulation results and discussion}

Traditional FDTD simulations [5] were employed to determine $E^{2}(f)$. They were performed for several sets of device shapes, specific resistances and frequencies (Fig. 2). As all other dimensions being equal, the average electric field amplitude within the sensor rises when specific resistance increases. For most sensors field $E$ substantially increases when frequency is growing. However, for the longest sensor $(l=0.6 \mathrm{~mm})$ with specific resistance $2 \Omega \mathrm{cm}$ a clear exception is observed. The increase of the average amplitude is noticed at lower frequency only, whereas in the frequency range of $98-118 \mathrm{GHz} E$ becomes nearly independent of frequency. As a result the dependence of the average amplitude on frequency for this particular sensor is much closer to the ideal sensor than others (Fig. 2 right).

To clarify the observed behavior, a more detailed study of resonant features of the sensor was performed. Results of calculations are shown in Fig. 3. As one can see, a sharp resonance peak is observed at $90 \mathrm{GHz}$ for high specific resistance sensor. A corresponding distribution of the electric field amplitude is presented on 

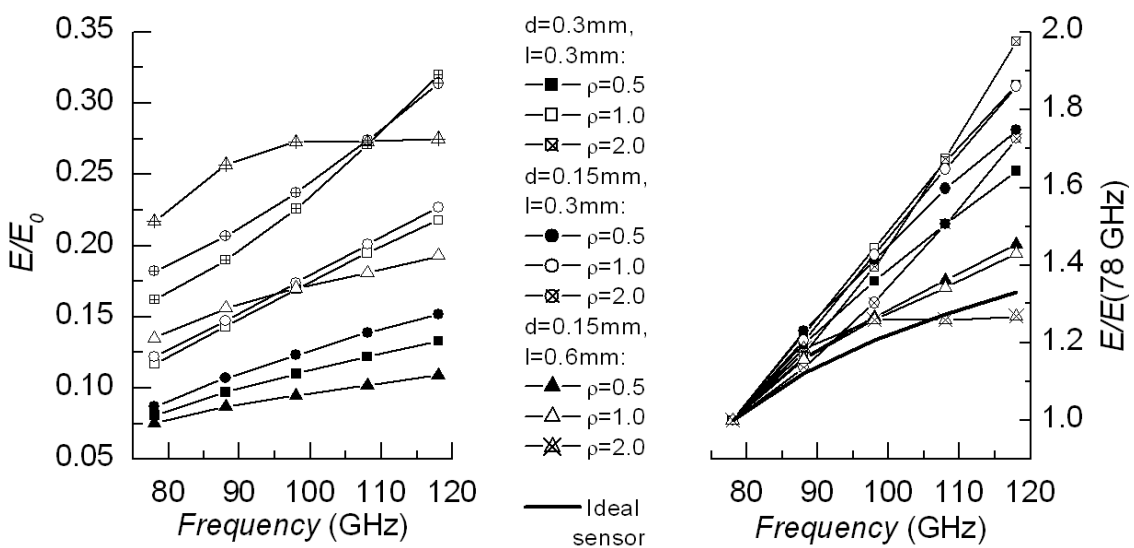

Fig. 2. Average electric field amplitude versus frequency for various shapes of the sensor and various specific resistances of semiconductor material. The amplitude is normalized to a value in the center of empty waveguide (left) and to a value of the average electric field amplitude within sensor at $78 \mathrm{GHz}$ (right). All simulations were performed for $h=0.1 \mathrm{~mm}$ sensor height. The legend of the figure displays dimensions $d, l$ of the sensor and specific resistances in $\Omega \cdot \mathrm{cm}$. Thick solid line indicates the desirable dependence (ideal sensor).
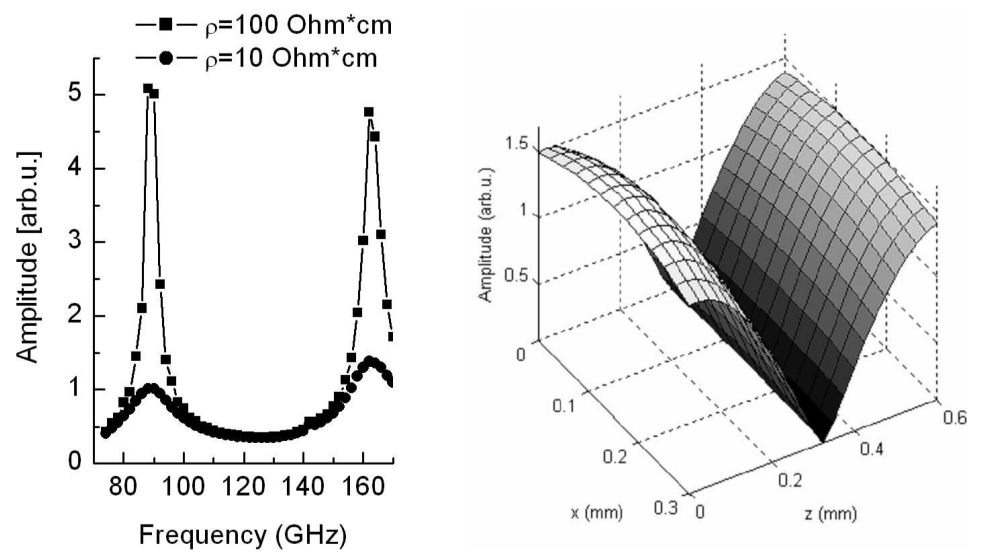

Fig. 3. Average amplitude of electric field in the RS versus frequency (left) and distribution of the electric field amplitude within the sensor at $90 \mathrm{GHz}$ (right). Simulations were performed for $d=0.15 \mathrm{~mm}, l=0.6 \mathrm{~mm}$ sensor. The legend indicates the specific resistance of the sensor for two cases.

the right part of the graph. The average electric field amplitude is dramatically reduced by increasing losses in the RS by decreasing its specific resistance. It seems that this resonance is responsible for observed dependence of $E$ on frequency for the RS made of $2 \Omega \mathrm{cm}$. The second resonance pike observed at $160 \mathrm{GHz}$ has little influence on average amplitude within the $78-118 \mathrm{GHz}$ range. 
In summary, our calculation has revealed that properties of the RS can be substantially improved by employing its resonant properties: by changing the resonance position in the frequency scale and controlling losses by selecting specific resistance. Therefore, the RS having nearly constant sensitivity in a waveguide's frequency range has been proposed. A similar design of the RS can be used for other frequency bands as well.

\section{Acknowledgment}

Authors would like to acknowledge the support of Lithuanian State Science and Studies Foundation through the program "Advanced Millimeter Wave Electronics".

\section{References}

[1] V.L. Bratman, A.E. Fedotov, Y.K. Kalynov, V.N. Manuilov, M.M. Ofitserov, S.V. Samsonov, A.V. Savilov, IEEE Trans. Plasma Sci. 27, 456 (1999).

[2] Yi Sheng Yeh, Chien Lun Hung, Chi-Wen Su, Ting-Shu Wu, You-Yan Shin, Yu-Tsung Lo, Int. J. Infrared Millim. Waves 25, 29 (2004).

[3] M. Dagys, Ž. Kancleris, R. Simniškis, E. Schamiloglu, F.J. Agee, IEEE Anten. Propag. Mag. 43, 64 (2001).

[4] V. Dienys, Ž. Kancleris, Z. Martūnas, Fiz. Tekh. Poluprovodn. 13, 1706 (1979).

[5] A. Taflove, Computational Electrodynamics: The Finite-Difference Time-Domain Method, Artech House, Norwood (MA) 1995 (and references within). 